\title{
V-Zn (Vanadium-Zinc)
}

\section{H. Okamoto}

The V-Zn phase diagram with three intermediate phases in [Massalski2] was redrawn from [1989Smi]. Among these phases, $\mathrm{V}_{4} \mathrm{Zn}_{5}$ and $\mathrm{VZn}_{3}$ were adopted from [1963Cha]. The third phase $\mathrm{VZn}_{16}$ was shown in the phase diagram, but its existence was questioned. Furthermore, another phase $\mathrm{V}_{3} \mathrm{Zn}$ had been reported to exist, but [1989Smi] concluded that this is not an equilibrium phase.

[2010Cha] confirmed the absence of both $\mathrm{VZn}_{16}$ and $\mathrm{V}_{3} \mathrm{Zn}$ by means of $\mathrm{X}$-ray diffraction analysis and scanning electron microscopy with energy-dispersive X-ray spectrometry for variously heat treated samples.

The V-Zn phase diagram calculated by [2010Cha] (Fig. 1) is in good agreement with experimental data of [1963Cha] $\left(<800^{\circ} \mathrm{C}\right)$.
V-Zn crystal structure data shown in Table 1 was adopted from [1989Smi].

\section{References}

1963Cha: M.G. Chasanov, R. Schablaske, P.D. Hunt, and B. Tani, The Zinc-Vanadium Phase Diagram. Trans. Metall. Soc. AIME., 1963, 227(4), p 485-488

1989Smi: J.F. Smith, V-Zn (Vanadium-Zinc), Phase Diagrams of Binary Vanadium Alloys, J.F. Smith, Ed., ASM International, Metals Park, OH, 1989, p 323-325

2010Cha: K. Chang, Y. Du, W. Sun, H. Xu, and L. Zhou, Thermodynamic Assessment of the V-Zn System Supported by Key Experiments and First-Principles Calculations. Calphad, 2010, 34(1), p 75-80

Table $1 \quad$ V-Zn crystal structure data

\begin{tabular}{lccccc}
\hline Phase & Composition, at.\% $\mathbf{Z n}$ & Pearson symbol & Space group & Strukturbericht designation & Prototype \\
\hline$(\mathrm{V})$ & 0 & $c I 2$ & $I m \overline{3} m$ & $\mathrm{~A} 2$ & $\mathrm{~W}$ \\
$\mathrm{~V}_{4} \mathrm{Zn}_{5}$ & 55.6 & $t I 18$ & $I 4 / m m m$ & $\ldots$ & $\ldots$ \\
$\mathrm{VZn}_{3}$ & 75 & $c P 4$ & $P m \overline{3} m$ & $L 1_{2}$ & $\mathrm{AuCu}$ \\
$(\mathrm{Zn})$ & 100 & $h P 2$ & $P 6_{3} / m m c$ & $\mathrm{~A} 3$ & $\mathrm{Mg}$ \\
\hline
\end{tabular}

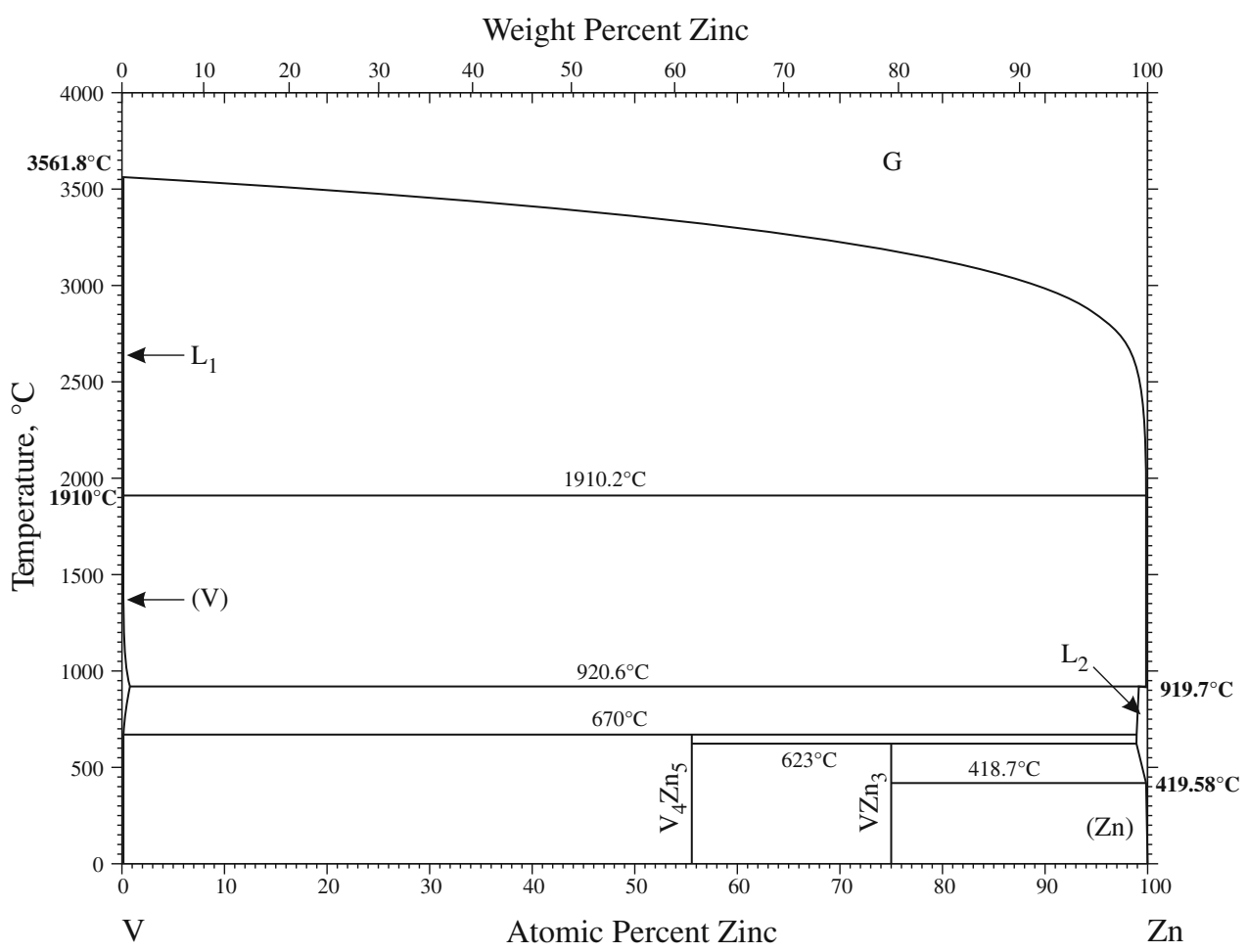

Fig. 1 V-Zn phase diagram 